\title{
Coulisses
}

Revue de théâtre

4 | Été 1991

Varia

\section{Présentation du théâtre nô}

\section{François Migeot}

\section{OpenEdition}

Journals

Édition électronique

URL : http://journals.openedition.org/coulisses/1691

DOI : 10.4000/coulisses. 1691

ISSN : 2546-9460

\section{Éditeur}

Presses universitaires de Franche-Comté

\section{Édition imprimée}

Date de publication : 1 juin 1991

Pagination : 52-65

ISSN : 1150-594X

\section{Référence électronique}

François Migeot, «Présentation du théâtre nô », Coulisses [En ligne], 4 | Été 1991, mis en ligne le 04 juillet 2017, consulté le 21 octobre 2019. URL : http://journals.openedition.org/coulisses/1691 ; DOI 10.4000/coulisses.1691

Ce document a été généré automatiquement le 21 octobre 2019

Coulisses 


\title{
Présentation du théâtre nô
}

\author{
François Migeot
}

1 Pour dissiper toute ambiguïté, il convient, d'entrée de jeu, de préciser que les ambitions de cet article restent bien modestes : il ne vise qu'à décrire, pour un public non averti, un mode d'expression théâtral qui demeure mal connu dans nos contrées où le théâtre à l'italienne reste la référence.

2 Je me contenterai donc de rédiger quelque chose comme la fiche technique du théâtre nô en m'appuyant sur les rares documents en français qui me soient accessibles ${ }^{1}$. Pour ce faire, je ne m'autoriserai que de moi-même, que de ma curiosité et des quelques connaissances qu'un séjour prolongé au Japon m'a apportées. Circonstances aggravantes, je ne suis pas homme de théâtre et ne puis me prévaloir d'aucun titre dans ce domaine.

3 Pareille légèreté, si elle peut passer pour scandaleuse aux yeux d'un certain purisme d'ici et de là-bas, ne me paraît cependant pas de mauvais aloi dès lors que je me propose de donner une image désacralisée et rafraîchie d'un genre dramatique recouvert d'une poussière quatre fois centenaire et souvent confiné par la tradition dans un rituel figé aux allures de cérémonial.

\section{Sources du nô}

Le poids écrasant de cette tradition vient sans doute du fait que le nô a été et reste entre les mains d'une aristocratie.

D'abord, aristocratie des cinq familles d'acteurs qui, de père en fils (puisque filles et femmes n'ont pas accès aux planches du nô), depuis le $\mathrm{xvI}^{\mathrm{e}}$ siècle (et pour certaines avant), monopolisent jalousement cet art : l'école Kanze, fondatrice, puis l'école Hosho, l'école Komparu, l'école Kongo, et l'école Kita. Chacune de ces dynasties se distingue des autres par un point particulier relatif à l'interprétation: mimiques, exacte fidélité au jeu ancien, plus ou moins grand raffinement dans l'interprétation..., et par un répertoire pouvant présenter des variantes, dans les textes, d'une école à l'autre. 
6 Ajoutons aussi que les deux protagonistes de toute pièce de nô, le Shite (personnage principal) et le Waki, ont été formés à des écoles différentes. Une hiérarchie plus ou moins explicite valorise les écoles de Shite au détriment de celles de Waki qui restent des personnages effacés malgré les efforts de certains auteurs pour revaloriser ce rôle. Au bas de l'échelle, viennent les écoles des acteurs de Kyôgen qu'on voit à l'œuvre dans les interludes bouffons servant d'entracte dans la succession des cinq nô qui formaient traditionnellement une journée.

7 Aristocratie aussi du public, ancien et contemporain. Le premier, au sens propre, puisqu'il s'agissait esentiellement d'un spectacle conçu, au plus haut niveau, à l'usage de la cour du Shogun ou de l'empereur, et au plus bas, à l'usage des aristocrates guerriers formant la classe des Samuraï. Le second, au sens figuré, puisqu'il s'agit d'une élite de lettrés connaisseurs qui, une fois mise à mal la féodalité, à la fin du XIX siècle, par l'ère Meiji, ont pris le relais du public naturel en se faisant les garants de la tradition et en remplaçant le privilège du sang par celui de l'initiation. En effet, on reconnaît dans les salles ces initiés, encore de nos jours, par le livret qu'ils suivent largement autant que le spectacle, et par lequel ils vérifient que le texte - en japonais du $\mathrm{XV}^{\mathrm{e}}$ ou $\mathrm{XvI}^{\mathrm{e}}$ siècle - n'a pas été accroché et que les déplacements des acteurs (schématisés en face du texte) sont bien en absolue conformité avec leurs tablettes.

8 Ainsi, cette tradition, faute d'un véritable renouvellement dans l'interprétation et dans le répertoire depuis les apports décisifs de Kan-ami et de son génial fils Zeami, aux XIV et $\mathrm{Xv}^{\mathrm{e}}$ siècles, finit par devenir un poids et faire du nô cette cérémonie quelque peu austère que nous évoquions et que René Sieffert, connaisseur et traducteur de Zeami, stigmatise sans gêne :

Dans cette atmosphère confinée et intemporelle, cet art vigoureux et populaire, né dans une atmosphère de concours et de compétition, s'assoupit tout doucement pour devenir un spectacle de musée, entretenu avec une religieuse componction par de pieux conservateurs officiant devant un parterre recueilli et somnolant. Nous savons, par des documents incontestables, que l'interprétation actuelle d'un nô prend en moyenne presque deux fois plus de temps qu'à l'époque des Ashikaga! C'est dire que cette lenteur que la plupart des auteurs occidentaux, et japonais, prennent pour le caractère essentiel du nô, ne lui était en aucune façon congénitale, et l'on constatera, en lisant Zeami, que celui-ci la condamnerait très certainement, s'il lui était donné d'assister à une représentation d'aujourd'hui... Apprécierait-il au moins l'austérité froide et la dignité revêche qui en résultent? Je n'oserais en jurer !

En effet, comme le rappelle à juste titre Sieffert, le nô n'a pas toujours été l'affaire de l'aristocratie, et par ses origines, au contraire, il renvoie à des sources bien souvent populaires. Ce n'est qu'en 1374 que le Shogun Yoshimitsu, de la dynastie des Ashikaga, après avoir été subjugué au cours d'une représentation par le jeu de Yuzaki Saburô Kiyotsugu (alias Kan-ami), le fit entrer à sa cour -contre l'avis de tous et au mépris de l'étiquette- avec son fils qui allait devenir le célèbre Zeami. En effet, le sarugagu, ancêtre du nô, dont le père de Zeami était l'un des plus illustres tenants, passait pour vulgaire et ne jouissait pas des faveurs de la cour, contrairement au dengaku qui avait les honneurs de l'aristocratie militaire de l'époque. Il faut donc attendre cette date pour que ce qui allait devenir le nô acquière droit de cité auprès des princes et qu'il gagne ses lettres de noblesse avec Zeami à la génération suivante.

10 Auparavant, la tradition du futur nô s'enracine dans divers courants dont les deux principales manifestations étaient le sangaku et le bugaku. 
11 Le bugaku vient de Chine et arrive à l'aristocratie nippone par l'intermédiaire de la Corée au $\mathrm{VIII}^{\mathrm{e}}$ siècle. Il consiste en des danses de cour accompagnées de musique (gagaku). Le style se japonise $\mathrm{du} \mathrm{IX}^{\mathrm{e}}$ au XII ${ }^{\mathrm{e}}$ siècle et influence même les danses shintô indigènes, les gakura, dans lesquelles Zeami veut voir une forme archaïque du nô. D'autre part, le sangaku vient lui aussi de Chine, mais son public est essentiellement populaire. Les «divertissements variés » qu'il offre s'apparentent aux spectacles de foire: funambules, jongleurs, montreurs d'animaux, de marionnettes, et autres saltimbanques, ce qui explique que ses adeptes eurent tôt fait de le rebaptiser sarugaku, c'est-à-dire « danses de singes ».

12 Toutefois, le sangaku comporte également un élément indigène, le dengaku qui est issu d'anciennes danses agrestes autochtones. C'est par cet élément que vont se manifester de nouvelles préoccupations esthétiques qui vont faire du dengaku un genre noble qui s'opposera bientôt au sarugaku. C'est à l'école du premier que Kan-ami va mettre le sarugaku, ouvrant ainsi la voie au nô, tandis que l'ancien répertoire comique issu du traditionnel Sarugaku deviendra celui du kyôgen.

\section{Le théâtre nô}

Caractériser le théâtre nô pour un public occidental n'est pas chose aisée car il ne ressemble en rien à ce que nous connaissons et à quoi nous pourrions nous référer. Procédons plutôt par négation : ce n'est pas seulement du théâtre puisqu'il inclut aussi le chant, la musique, la danse et le mime. Ce n'est pas un théâtre d'action puisqu'aucune intrigue ne se déploie au cours de la pièce; tout au plus s'agit-il d'évoquer par un récit une action passée et d'en mimer ou danser certains passages en contrepoint de la narration. Le nô est donc à mi-chemin entre le drame et le poème épique. Il ne s'agit pas non plus d'un théâtre religieux, encore que le Bouddhisme y soit fortement présent, mais plutôt d'un spectacle qui trouve matière à mettre en scène une forme de beauté à travers l'occasion que peut offrir telle ou telle référence religieuse. Il n'est pas davantage un théâtre de caractères : avec les personnages du nô, bien plus qu'à des individus dont la représentation se voudrait réaliste, on a affaire à des types (le guerrier, le bonze, la femme, le vieillard...) ; aucune interaction n'est là pour permettre que se dégage une psychologie : l'un des personnages permet simplement à l'autre de raconter son histoire. Qui plus est, la notion même de personnage approche souvent la dissolution dans la mesure où le même texte est pris en charge successivement par les deux acteurs et par le chœur, si bien que le sujet de renonciation tend à s'évanouir dans un discours flottant.

14 Faute de mieux, il faut donc se résigner à reprendre, avec la même insatisfaction que lui, la formule que propose R. Sieffert pour cerner le nô : «Long poème chanté et mimé, avec accompagnement orchestral, généralement coupé par une ou plusieurs danses qui peuvent n'avoir aucun rapport avec le sujet. » 


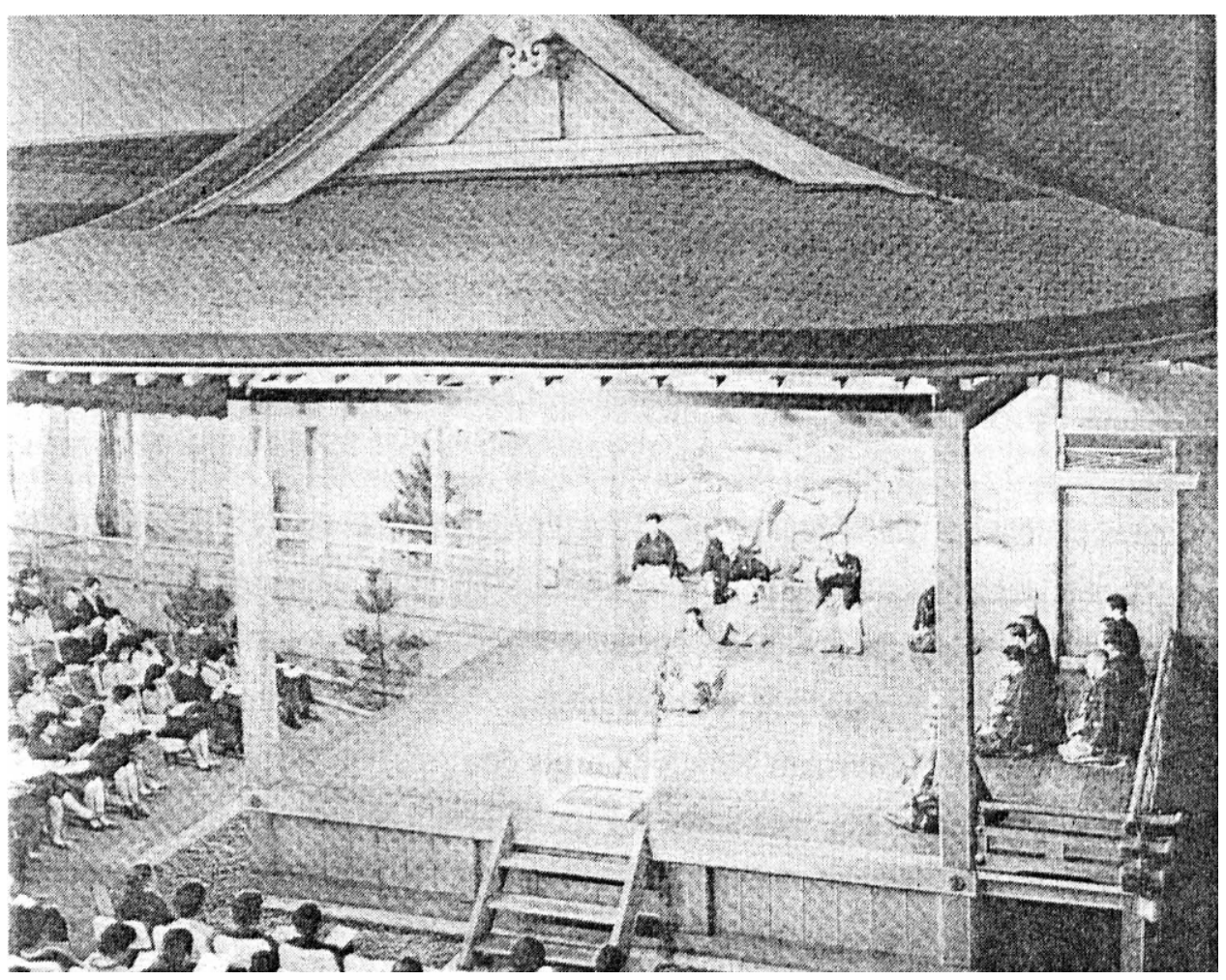

Planche 1. Vue d'ensemble de la scène d'un théâtre nô.

\section{La scène}

Depuis un siècle environ, le nô a quitté les cours des temples où il était donné en plein air. Mais dans les salles actuelles il garde encore, par la toiture notamment, les marques de ses anciens séjours (voir la planche $\mathrm{n}^{\circ} 1$ ).

La scène comporte un plateau (butaï) en bois blanc, carré, de trois ken (un peu moins de $6 \mathrm{~m}$ ) de côté, posé sur sept grandes jarres qui permettent une meilleure résonnance des appels du pied. Du toit tombent en ses coins quatre piliers (voir planche $\mathrm{n}^{\circ} 2$ ) qui délimitent l'espace : le pilier du Shite (shite-bashira), celui du Waki (waki-bashira), celui de la flûte (fue-bashira), et enfin le metsuke-bashira qui sert de pilier de repère aux acteurs masqués. Partant du fond de la scène vers la gauche, une galerie légèrement pentue (hashi-gakari), le "pont», mène aux coulisses, la «salle du miroir » (gakuya), dont elle est séparée par un rideau. Quand un acteur entre en scène, il emprunte ce couloir bordé de trois pins successifs qui lui servent de marques s'il est masqué, et il apparaît ainsi progressivement au public avant d'atteindre le plateau. Au fond de la scène, comme toile de fond, est peint au mur l'arbre emblématique du nô, un vieux pin sombre, devant lequel vont prendre place l'orchestre et les « surveillants » (kôken) qui sont chargés de remplacer un éventuel acteur défaillant.

17 Sur la droite, en bordure du carré du plateau, entre le pilier de la flûte et celui du Waki, se tient, sur deux rangs, le chœur qui compte jusqu'à douze personnes. L'ensemble de la scène est entouré de galets et de sable blanc qui devait jadis servir de réflecteur à la lumière solaire. 


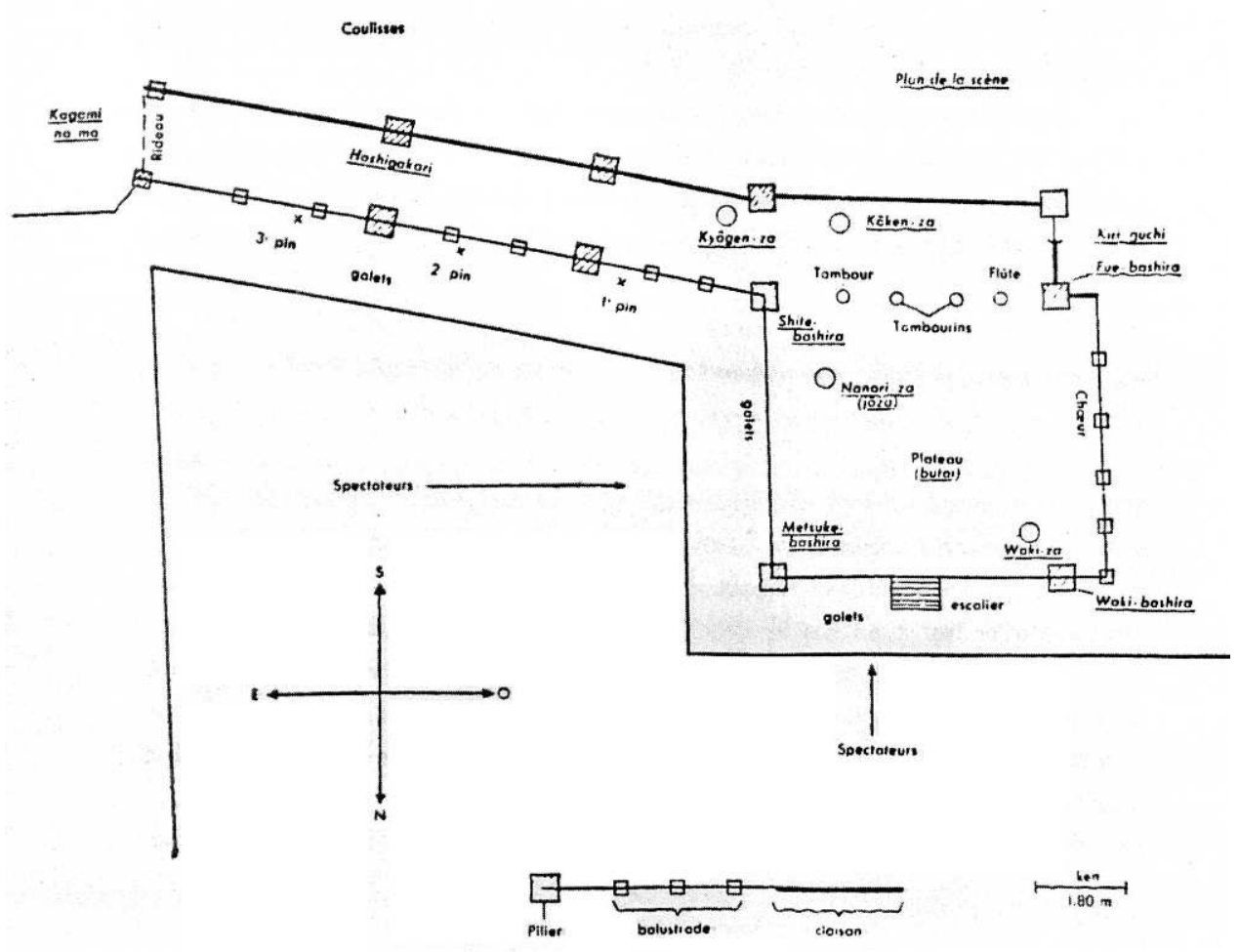

Planche 2.

\section{Chœur et musiciens}

Choristes et musiciens sont les premiers à entrer en scène ; ils sont aussi les derniers à la quitter. Ils portent le costume « de ville » de l'époque des Tokugawa (1600-1868), en soie noire.

Le flûtiste est le premier des musiciens à entrer. Il s'installe près de son pilier. Son instrument est une flûte traversière en bambou qui rend des sons riches en harmoniques. Il est suivi de deux tambours, un grand et un petit, dont la peau peut être plus ou moins tendue par la pression du bras, ce qui leur permet de rendre un son modulé.

Les musiciens accompagnent leur jeu de cris brefs ou filés, avec un timbre de voix rauque très particulier pour une oreille non habituée. La musique qu'ils exécutent a d'abord une fonction d'ouverture dans le spectacle, puis, une fois les acteurs en scène, elle ponctue le chant et rythme la danse plus qu'elle ne les accompagne. Quant au chœur, chantant à l'unisson, il relance le récit de l'acteur, commente ses actions ou effectue des descriptions, (voir planche 3). 


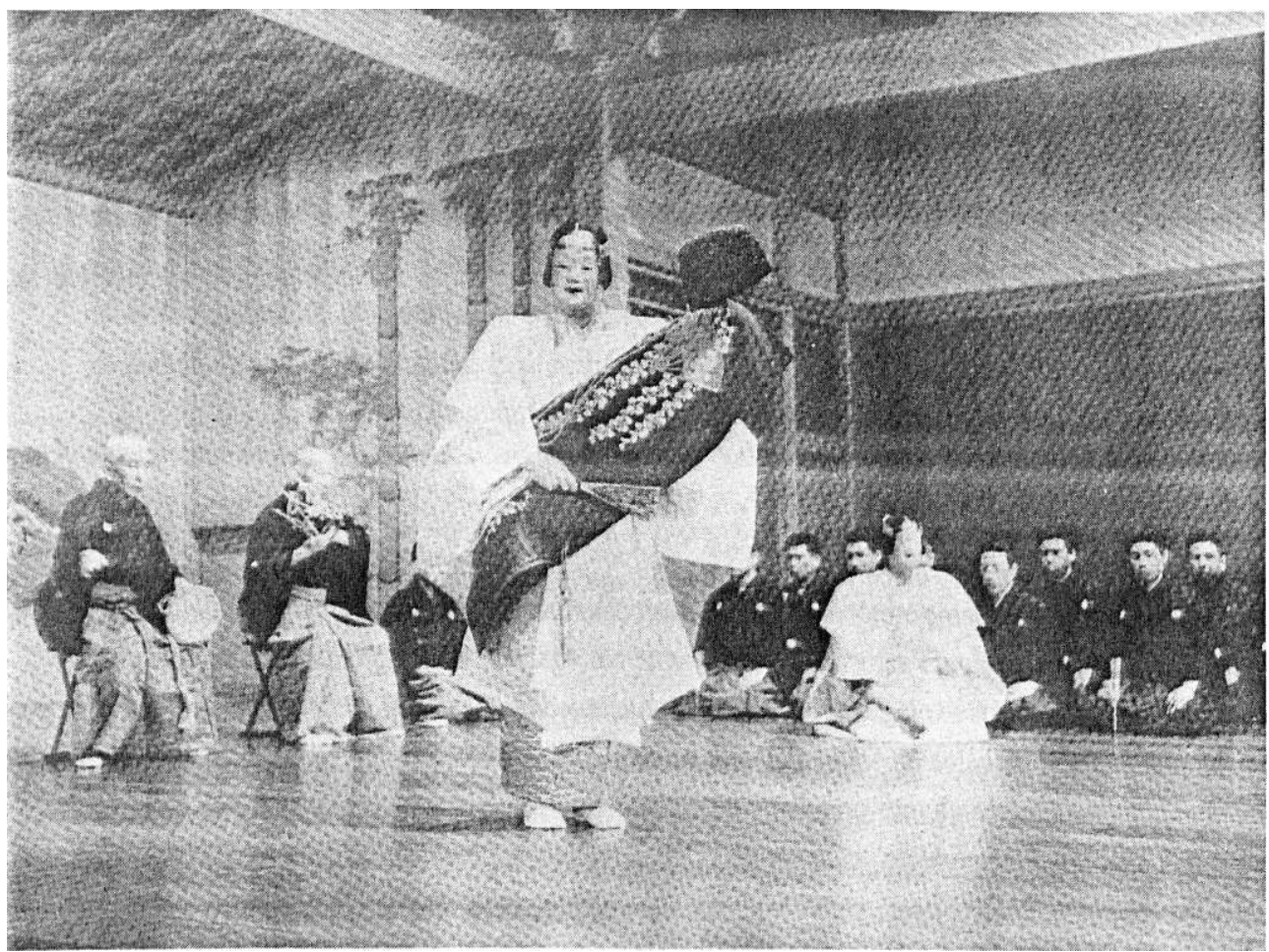

Planche 3. Une scène de Matsukaze, pièce nô typique.

\section{Les acteurs}

21 Le premier personnage à rentrer sur scène est presque toujours le Waki (« celui qui est à côté »). Le plus souvent, c'est un moine voyageur qui se présente, expose les buts de son voyage et va s'asseoir près de son pilier dont il ne se lèvera que pour d'éventuelles actions d'exorcisme. Il joue à visage découvert, il déclame et ne chante pas; il ne danse pas non plus. Il est là pour susciter la venue du Shite (« celui qui agit ») qui apparaîtra souvent d'abord sous des apparences ordinaires (Mae-shite), pour réapparaître ensuite (Nochi-jite), dans la deuxième partie, sous sa véritable nature : démon, esprit, dieu...

22 Le Shite déclame, chante, danse et monopolise tout l'intérêt du spectacle dont le Waki n'est lui-même qu'un témoin ou un médium. Il est somptueusement habillé (même s'il est censé représenter une personne de condition modeste), porte presque toujours le masque et une perruque symbolisant le personnage qu'il incarne: homme jeune, vieillard, guerrier, jeune femme, vieille femme, démon ou divinité (voir planche 4). Waki et Shite peuvent parfois être accompagnés de suivants (tsure) ou de compagnons (tomo).

23 Ajoutons à cela l'intervention d'une troisième catégorie d'acteurs, le Kyôgen qui intervient souvent à l'intérieur de la pièce entre la première et la seconde partie (aikyôgen) pour faire un récit et permettre au Shite de changer de costume. Par ailleurs, il intervient aussi avec ses pairs, entre deux nô, pour exécuter une farce (Kyôgen) destinée à détendre l'atmosphère. 


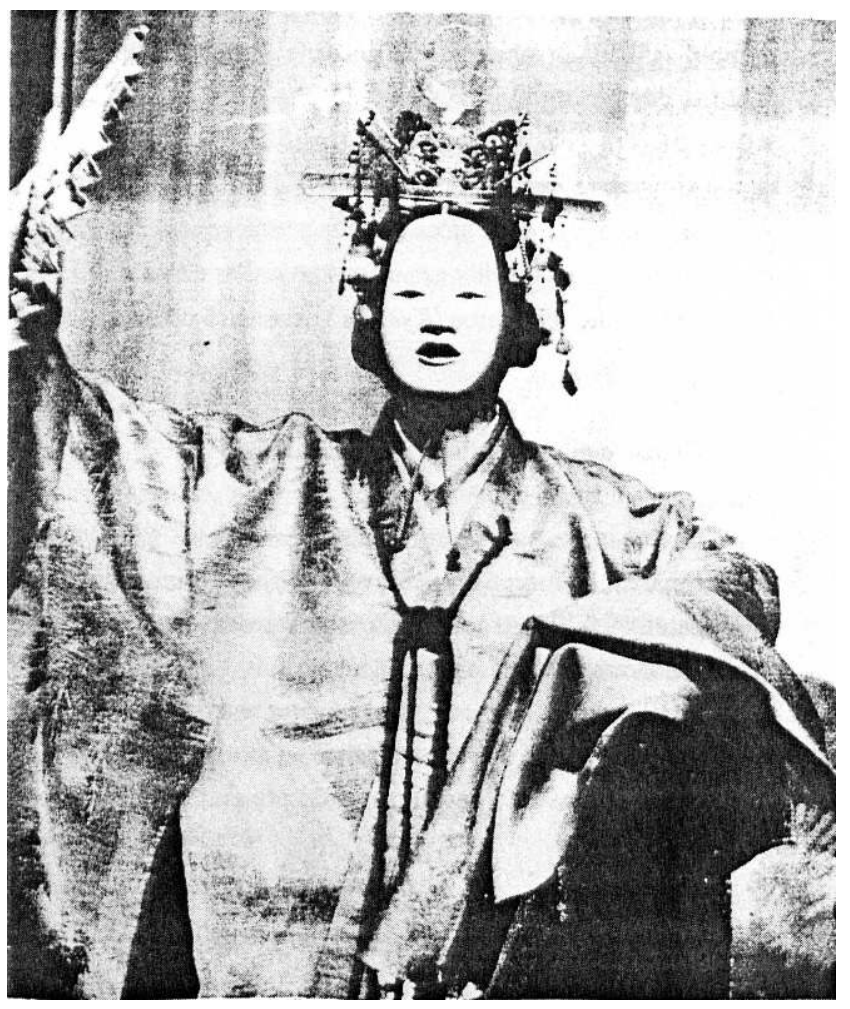

Planche 4. Personnage principal.

\section{Costumes, masques, accessoires}

Les costumes, toujours très riches pour le Shite, sont tous très stylisés, car plutôt qu'un individu, c'est à un type humain (ou divin) qu'ils renvoient. Ils ne signifient donc que l'âge, le sexe, et le statut social. Pour le Waki, il s'agit presque toujours d'un costume sombre de moine et d'une coiffe en forme de mitre.

Le port du masque est exclusivement réservé au Shite (sauf si le waki représente un personnage féminin) et manifeste une appartenance au monde des esprits. Le masque conditionne fortement le jeu de l'acteur. Il ne s'agit pas pour lui de prétendre au réalisme mais à des physionomies stylisées.

L'expressivité n'est pas exclue pour autant car le jeu avec le masque, combiné à la gestuelle de l'acteur et aux variations de la lumière, permet de réaliser une grande variété de nuances.

27 Souvent d'une inestimable valeur artistique, le masque est en bois, plus petit que le visage de l'acteur, ce qui déréalise sa silhouette, par opposition à celle du Waki qui reste plus lourde, plus humaine; il a tendance à étouffer la voix de l'acteur ce qui l'oblige à avoir cette diction si caractéristique.

En ce qui concerne les accessoires, le théâtre nô est d'une sobriété remarquable. Aucun décor ne vient camper le heu, et fort peu d'objets viennent soutenir le jeu des personnages : un éventail qui, nous le verrons, soutient les mimiques et accompagne les danses, et parfois, pour esquisser des indications de lieu ou d'objet (temple, montagne, forêt, barque, char à bœufs...), quelques tiges de bambou et quelques pièces d'étoffe 
font l'affaire. De la même manière allusive, le sabre ou l'arc évoqueront le guerrier, une canne évoquera un vieillard ou un aveugle.

\section{Mimiques, jeu, mise en scène}

La mise en scène, au sens où nous l'entendons, n'existe pas pour le nô. Tout est programmé jusqu'au moindre geste de telle sorte qu'on a pu croire que la liberté de l'acteur était à peu près inexistante et que la perfection du jeu résidait dans l'exacte reproduction d'un modèle idéal figé depuis le $\mathrm{xvI}^{\mathrm{e}}$ siècle. Cette conception, assez majoritaire, mérite néanmoins d'être questionnée, comme nous le verrons.

Le nô comporte deux sortes de gestes, les uns motivés, ayant une signification conventionnelle, les autres, non motivés, purement choré-graphiques.

Parmi les premiers, on trouve par exemple le geste de la prière par lequel l'acteur joint le bout des doigts sur la poitrine, le « demi-pleur » (kata-shiori) où il pose la main sur les yeux, le "grand pleur » (moro-shiori), le geste de makura-no-oogi qui évoque, par la tête penchée et l'éventail devant la poitrine, la honte ou le sommeil, ou encore, le yuuken ("grand saut ») dans lequel l'acteur, tenant l'éventail ouvert devant la poitrine, le lève deux fois en signe de violente émotion, décision, ou grande joie.

Le jeu de l'acteur ne consiste donc pas à donner une vision réaliste du personnage mais plutôt à en donner une image essentielle. Zeami condamne tout ce qui, par la gestuelle, compromettrait la valeur de signe que doit acquérir le personnage, ce qu'il résume par le précepte de "mouvoir l'esprit aux dix dixièmes, mouvoir le corps aux sept dixièmes ». On retrouve encore cette idée lorsqu'il parle des quelques rôles où l'acteur doit jouer à visage découvert. Dans ce cas, dit-il,

il est indispensable que l'on adapte sa mimique au caractère de chaque personnage.

Mais, alors qu'il n'y a aucune nécessité d'imiter jusqu'à l'expression du visage, il

arrive pourtant que, modifiant son expression habituelle, l'on compose son visage.

C'est là un spectacle absolument intolérable. C'est par son comportement, par son allure qu'il faut imiter son personnage.

3 Toutefois, cette stylisation, cette épuration du personnage ne peut s'atteindre par l'imitation des maîtres consacrés, car elle doit être sans cesse recréée de l'intérieur par l'acteur, et non pas de l'extérieur par copie, faute de quoi l'acteur tombe dans le «style impersonnel » :

D'une manière générale, en matière de danse et de chant, tant que vous reproduisez ce que vous avez appris [...], votre style reste impersonnel. Je m'explique : même si votre jeu donne dans l'ensemble l'impression d'une bonne reproduction de ce que vous avez appris, vous n'en avez pas encore fait votre chose [...], votre nô ne s'élève pas.

On peut donc légitimement se demander si la conception qui accrédite l'idée que le nô a atteint la perfection au $\mathrm{XVI}^{\mathrm{e}}$ siècle, et que depuis lors on ne peut que reproduire religieusement cette perfection, n'est pas contraire à l'esprit même que promeut Zeami. En effet, on relirait avec profit, en pensant à ce « spectacle de musée, entretenu avec une religieuse componction par de pieux conservateurs» dénoncé par Sieffert, ce passage où Zeami critique les jeunes acteurs :

[...] Ils imitent sans étudier. Si, passant des deux éléments aux trois types ${ }^{2}$ ils se formaient au cycle des exercices par âge ${ }^{3}$, s'ils suivaient un entraînement progressif et continu, ils pourraient parvenir à la maîtrise dans tous les domaines et se constituer un style personnel ; or ils se contentent d'imiter ce qu'ils voient faire [...]. 
D'autre part, le nô, tel qu'il est arrivé jusqu'à nous, semble fixé de manière éternelle pour un public uniforme et atemporel. C'est, là encore, peu conforme à la grande attention pour chaque public que recommande Zeami, et qui caractérisait le grand acteur qu'était son père :

[...] c'est pourquoi feu mon père, fût-ce dans quelque coin de campagne, ou dans un village de montagne écarté, exerçait son art en tenant compte de la disposition d'esprit de son public, et en attachant une importance capitale aux coutumes des lieux.

Attention au public qui se manifestait encore dans l'intuition que devait avoir l'acteur du moment juste pour prononcer les premières paroles à son entrée en scène :

Lorsqu'on rentre en scène pour un sarugaku, il est un moment précis pour entonner le sashikoto ou l'issei. Le devancer est mauvais, le laisser passer serait mauvais aussi. Donc, vous sortirez du foyer des acteurs, vous vous avancerez sur le pont et vous vous $\mathrm{y}$ arrêterez, vous regarderez dans toutes les directions et à l'instant même où le public, unanime dans l'attente, se dira : « là, il va entonner ! » vous entonnerez. Cela, [...] c'est l'intuition correcte du moment propice.

Enfin, pour clore ce sujet, mentionnons encore un point essentiel qui confère sans doute au nô actuel son "austérité froide» et cette «dignité revêche» dont parle Sieffert : les acteurs contemporains ne sont plus créateurs. Il y a quatre cents ans ou plus qu'on ne produit plus de nô et que les écoles ressassent le même répertoire. Or le véritable acteur est, selon Zeami, celui qui est aussi créateur de nô, Cette liberté qu'il n'a pas dans l'exécution de ce qui est écrit et fixé, il la regagne dans la conception de pièces qu'il écrit pour lui :

Quand l'auteur d'un nô est un autre que l'interprète, quelque habile que soit ce dernier, il n'est pas libre d'interpréter comme il l'entend. Quand la pièce est de sa propre composition, paroles et comportements sont à sa discrétion. [...] L'acteur qui ne disposerait pas de nô bien à lui est pareil au guerrier qui, fût-il homme à tenir tête seul contre mille, partirait en campagne sans armes.

Voilà donc pourquoi, dans le domaine de l'interprétation, Sieffert espère un salutaire retour à Zeami :

Si les acteurs voulaient bien [...] revenir aux sources et secouer la poussière des siècles, il se pourrait bien que le nô retrouve une seconde vie, aussi riche que la première, et, qui sait, des sources d'inspiration longtemps taries. [...] Se pourrait-il qu'on lise de nouveau Zeami dans les écoles de nô?

\section{Répertoire, organisation du spectacle}

Le répertoire compte environ 240 pièces qui se répartissent en cinq catégories pour ce qui est de leur sujet. Les allusions à la saison contenues dans les pièces imposent qu'on les joue à l'époque de l'année qui convient. Une journée de nô comportait traditionnellement un enchaînement de cinq pièces séparées par quatre interludes de kyôgen. De nos jours, on donne rarement plus de deux pièces et un kyôgen. Cela ne va pas sans briser l'harmonie recherchée par la combinaison des cinq nô à travers l'application du principe fondamental jo, ha, $k y u$ (« ouverture, développement, finale »).

Les pièces de la première catégorie, souvent les plus anciennes, "pièces de dieux " (kami-mono), ne passent pas pour être les meilleures; leur fonction est surtout de mettre en condition le spectateur pour une journée. 
41 Ensuite viennent les "pièces de guerriers " (shura mono), premières du registre ha, parmi lesquelles on trouve plusieurs chefs-d'œuvres de Zeami (Kaneshira, Sanemori). Elles obéissent toutes au même schéma : un moine voyageur (le Waki) fait halte sur un ancien champ de bataille; il rencontre un vieillard (Mae-shite) qui lui conte les hauts faits d'un guerrier mort au combat. Le moine passe la nuit en prière sur les lieux; soudain le spectre revient (Nochi-jite) sous les traits d'un guerrier mort au combat et revit, comme pour les exorciser, les circonstances de sa mort. Le moine le délivre de ce passé ou échoue et le laisse retomber dans son enfer.

On donne ensuite une "pièce de femme" (Onna-mono), propre entre toutes à faire éclore le «charme subtil » cher à Zeami. Le schéma est le même que pour la catégorie précédente, mais transposé au féminin: le moine rencontre en première partie une femme, puis l'esprit qu'il verra apparaître en seconde partie sera celui de l'héroïne défunte. Toutefois, il est des pièces où le Shite de la première et de la seconde partie représente une femme vivante et contemporaine du Waki; tel est le cas, par exemple, de Yuya. Les héroïnes de ces pièces sont parfois tirées des plus belles figures du Genjimonogatari, telle Yûgao. Ces pièces passent pour être les plus représentatives de l'art du nô.

Viennent ensuite les pièces de la quatrième catégorie, qui clôturent la série ha, et qui appartiennent au «monde réel ». Le Shite ne porte donc pas de masque. Dans ces pièces, «pièces de folles » (Monogurui-mono), le Waki devient un partenaire à part entière du Shite, ce qui rend ces pièces moins difficiles à suivre pour un non-initié. L'argument le plus fréquent est le suivant : une femme perd l'esprit à la suite de la disparition d'un enfant ou d'un mari. Elle erre à sa recherche jusqu'à retrouver la raison avec l'être aimé. Parfois, elle ne trouve qu'une tombe et sombre alors dans la démence.

44 Enfin, pour clore la journée, on trouve les pièces de l'élément kyû, rapides et animées, pièces souvent «de démons » (Oni-mono). Le moine rencontre une personne (vieillard ou jeune femme) qui se révèle ensuite être un démon. Dans la dernière partie, le Shite exécute une danse véhémente, appelée hatakari, et qui s'oppose à d'autres danses du type mai.

\section{Le Kyôgen}

Entre chacun de ces cinq nô, on aura eu le soin d'intercaler des kyôgen. Ces farces, méprisées par les puristes, ont le mérite de rétablir un équilibre, après la tension du nô, par l'humour et la parodie. Le rire qu'ils sollicitent n'est pas, il est vrai, toujours des plus fins, et les personnages en scène viennent de la tradition populaire : l'ivrogne, le moine paillard, le valet rusé, le maître brutal, l'avare, la femme jalouse... À noter, aussi, certains kyôgen qui sont une parodie de nô. Les acteurs (toujours masculins ici aussi) sont alors masqués et donnent une caricature des grands sujets du répertoire « sérieux ». 


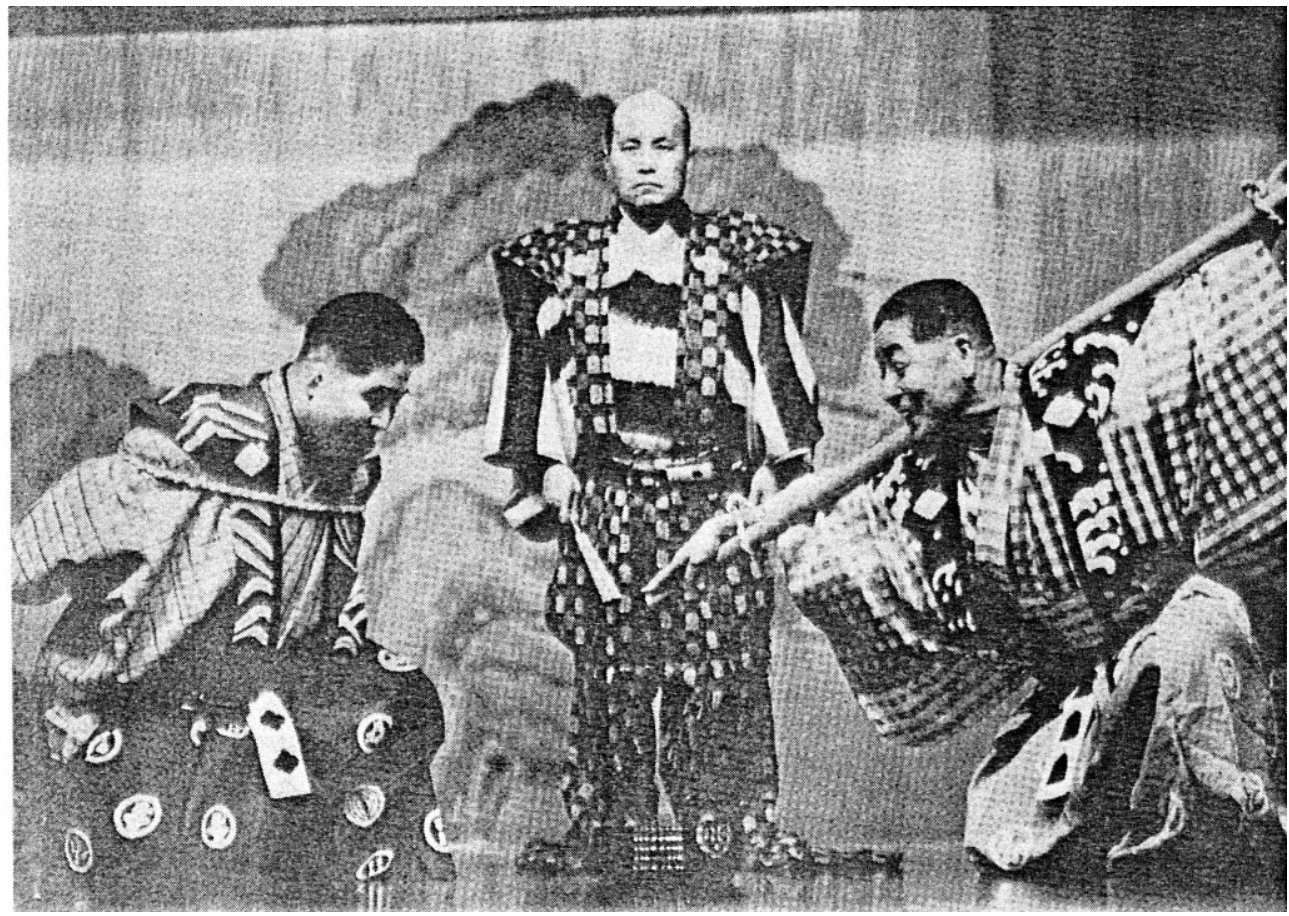

Une scène de Kyôgen.

\section{Structure d'une pièce de nô}

Le principe jo, ha, kyû qui gouverne l'ensemble d'une journée de nô régit également chaque pièce, puis, à l'intérieur de chacune d'elles, chacune de ses parties. En fait, cet équilibre se retrouve à tous les niveaux du théâtre nô : du niveau macroscopique de la journée au niveau microscopique de la phrase, en passant par les pas de danse, la marche ou la séquence instrumentale.

Dans la première partie d'une pièce, on compte un élément jo et un élément ha. L'élément jo comporte une ouverture instrumentale pendant laquelle le Waki se rend au nanori-za. Là, il entonne un chant d'introduction (shidai), puis se présente en déclamant (nanori). Il exécute ensuite un chant de voyage (michiyuki) puis opère, en déclamant de nouveau, une description du lieu (tsuki-serifu).

L'élément ha comporte une séquence jo: c'est l'entrée du Shite sur le pont (issei no hayashi) accompagné de l'orchestre. Il s'arrête sur le pont et entonne un chant d'introduction (issei). Il se dirige vers le plateau en chantant et s'arrête au jo-za.

Puis une séquence ha: c'est la rencontre du Waki et du Shite. Ils dialoguent face à face, immobiles, relayés par le chœur. Ensuite un dernier dialogue a lieu entre le Waki et le Shite auquel le chœur se mêle, puis le mae-shite quitte la scène.

À la suite de cette première partie intervient l'interlude ai-kyôgen où le kyôgen (incarnant un habitant du lieu) raconte au Waki, en langage simple et clair, ce que le Shite vient d'évoquer de manière confuse et troublée.

51 Vient ensuite la seconde partie, kyû, d'abord avec un élément jo où le Waki se lève et exécute un chant d'attente (machi-uta), ensuite avec un élément ha introduit par une partie instrumentale où le Shite, transformé (nochi-jite), fait son entrée; il chante un nouveau couplet d'introduction (sashi- uta). Il dialogue avec le Waki et il danse (mai ou 
hatakari selon les pièces), accompagné par le chœur et l'orchestre. Enfin vient le dernier élément de la seconde partie, élément kyû où le chœur entonne un dernier chant conclusif (kiri).

Telle est, grosso modo le schéma des nô classiques.

Voilà donc ce que pouvait se risquer à dire un amateur sans trop - espérons-le encourir les foudres des spécialistes. On regrettera sans doute que cet article ait été trop descriptif ; c'est vrai, mais je n'avais rien promis d'autre. Pour ceux des lecteurs qui resteraient sur leur faim, je ne peux que les engager à lire les traités et quelquesunes des pièces de Zeami qui sont maintenant accessibles en français et qui leur permettront, petit à petit, par l'éthique de l'acteur et l'esthétique du spectacle que le maître y esquisse, de pénétrer les Arcanes du nô.

\section{BIBLIOGRAPHIE}

Outre la traduction des traités de Zeami et de quelques-unes de ses pièces, outre les commentaires de leur traducteur, R. Sieffert largement cités ici, on lira avec profit, en français :

Noël Peri : Le Nô, Maison Franco-Japonaise, 1944.

Gaston Renondeau : Nô, Maison Franco-Japonaise, 1954.

Gaston Renondeau : Le Bouddhisme dans les Nô, Maison Franco-Japonaise, 1950.

\section{NOTES}

1. L'essentiel de ma documentation est puisé dans les conunentaires et la traduction par René Sieffert du livre de Zéami : La Tradition secrète du nô (Gallimard/Unesco, Connaissance de l'Orient, 1960). Un utile complément d'information m'a parfois été apporté par les conférences sur le nô que le professeur Chujo avait données en 1987 à l'université de Franche-Comté. Pour une bibliographie générale, voir en fin d'article.

2. Les deux éléments fondamentaux pour Zeami sont le chant et la danse., et les trois types de base sont le vieillard, la femme, le guerrier.

3. Zeami distingue sept tranches d'âge chez le comédien, de sept ans à cinquante et plus... 